\title{
EFEITOS DA ELETROANALGESIA SOBRE A DOR E FORÇA MUSCULAR RESPIRATÓRIA EM PACIENTES NO PÓS- OPERATÓRIO DE CIRURGIA BARIÁTRICA
}

\section{EFFECTS OF ELETROANALGESIA ON PAIN AND RESPIRATORY MUSCULAR FORCE IN PATIENTS IN THE POSTOPERATIVE SURGERY OF BARIATRIC SURGERY}

\author{
Cesar Antonio Luchesa ${ }^{1 *}$, Rafael Malta Dos Santos Barboza²
}

${ }^{1}$ Fisioterapeuta Mestre em Ciências da Saúde, Coordenador do serviço de Fisioterapia do Hospital São Lucas FAG, Professor do Centro Universitário FAG, ${ }^{2}$ Fisioterapeuta

*Autor correspondente: cesar.luchesa@fag.edu.br, https://orcid.org/0000-0003-1801-0277

DOI: $10.35984 / f j h . v 2 i 1.114$

\section{RESUMO}

As mudanças dos hábitos de vida e a alimentação rica em carboidratos são partes de um contexto de mudança cultural que levaram a uma epidemia de obesidade. $O$ tratamento convencional muitas vezes não surte efeito, sendo necessária intervenção cirúrgica. Este trabalho busca avaliar o efeito da eletroanalgesia (tens) convencional no quadro de dor e consequentemente na dor e força muscular respiratória em indivíduos no pós-operatório de cirurgia bariátrica. Trata-se de um ensaio clínico não controlado. Foram randomizados 10 indivíduos entre 18 e 50 anos no pós-operatório imediato de cirurgia bariátrica em um hospital do oeste do Paraná. Os participantes foram avaliados antes e após a aplicação terapêutica. Para aplicação foi utilizado o aparelho tens, convencional com frequência de $80 \mathrm{hz}$ e $\mathrm{T}=200 \mathrm{us}$ durante 30 minutos em uma única aplicação. Participaram deste estudo 10 pacientes, sendo $1(10 \%)$ masculino e 9 (90\%) feminino, com média de idade de 39,6 $\pm 11,78$. Referente a dor observou-se uma redução no pós-intervenção $(2,2 \pm 1,98)$ quando comparado no préintervenção $(5,6 \pm 1,57)$ com $P=0,000$. Os valores de pimáx e pemáx não apresentaram valores estatisticamente significativos com $p>0,05$. Conclui-se que o tens é uma ferramenta eficaz na redução da dor no pós-operatório de gastroplastia, sem efeito sobre a força muscular respiratória. Palavras-chaves: Estimulação Elétrica Nervosa Transcutânea, dor abdominal, fisioterapia.

\begin{abstract}
Changes in lifestyle and the carbohydrate-rich diet are part of a context of cultural change that has led to an obesity epidemic. Conventional treatment often has no effect, requiring surgical intervention. This study aims to evaluate the effect of conventional electroanalgesia (tens) on pain and consequently on pain and respiratory muscle strength in individuals after bariatric surgery. This is an uncontrolled clinical trial. Ten individuals between 18 and 50 years of age were randomized in the immediate postoperative period of bariatric surgery in a hospital located in the west of Paraná. Participants were assessed before and after therapeutic application. For the application, it was used the apparatus tens, conventional with frequency of $80 \mathrm{hz}$ and $T=200 u$ s during 30 minutes in a single application. Ten patients participated in this study, being 1 (10\%) male and 9 (90\%) female, with a mean age of $39.6 \pm 11.78$. Concerning pain, a post-intervention reduction $(2.2 \pm 1.98)$ was observed when compared in the pre-intervention $(5.6 \pm 1.57)$ with $P=0.000$. The pimax and pmax values did not present statistically significant values with $p>0.05$. Thus, it is concluded that tension is an effective tool in the reduction of postoperative pain of gastroplasty, with no effect on respiratory muscle strength. Keywords: Transcutaneous Electric Nerve Stimulation, Abdominal Pain, Physical Therapy Specialty.
\end{abstract}




\section{INTRODUÇÃO}

Segundo a Sociedade Brasileira de Cirurgia Bariátrica e Metabólica (SBCBM), a ocorrência de cirurgias bariátricas eletivas realizadas no Brasil entre 2012 e 2017, teve um aumento de 46,7\% (BATTISTELLI, 2018). Quando o tratamento convencional não surte efeito se faz necessárias terapêuticas mais agressivas, e neste contexto o tratamento mais eficaz para obesidade mórbida é a cirurgia bariátrica. Sua realização tem como objetivo principal melhorar a qualidade de vida para, assim, aumentar a expectativa de vida do obeso, resolvendo as desordens físicas e psicossociais que a obesidade acarreta (BUCHWALD et al, 2004).

Para a realização do procedimento cirúrgico pode-se usar diversas técnicas, entretanto o método Capella vem demonstrando sua prevalência (FANDIÑO et al, 2004) por apresentar um índice de morbidade baixo, ser considerada segura e com alto índice de perda peso (ORIA, 1998).

O procedimento cirúrgico gera lesão tecidual, seja pela manipulação cirúrgica direta visceral através da própria incisão ou pelo uso de afastadores cirúrgicos que fazem a exposição do campo operatório, isso faz com que o paciente apresente um quadro doloroso no período pós-operatório (TONELLA et al, 2006).

Mascarenhas (2014) em revisão de literatura, composta por 19 estudos sobre a fisioterapia em pacientes no pós-operatório (PO) de cirurgia abdominal alta (CAA) assim como a descrição das possíveis complicações, demonstraram o quadro doloroso como um fator de influência negativa na evolução do PO mesmo com a administração de medicamentos, isso provoca uma alteração da mecânica respiratória e limitação do trabalho diafragmático levando a uma respiração superficial.

$O$ fisioterapeuta atua com pacientes que estão em período PO abdominal, através de manobras de higiene brônquica, deambulação precoce e fortalecimento da musculatura respiratória, fazendo com que assim haja uma melhora da ventilação para então poder prevenir as complicações pulmonares (TREVISAN, 2010), porém a dor é vista como um fator limitante para a realização de procedimentos fisioterapêuticos precocemente (TONELLA et al, 2006), observando assim a importância de uma intervenção que vise a redução do quadro doloroso.

Com o aumento da incidência de cirurgias bariátricas realizadas atualmente, as complicações descritas, destacando o nível de dor apresentado no período pósoperatório e as ferramentas disponíveis como fonte de analgesia utilizadas por fisioterapeutas, este estudo tem a finalidade de avaliar a eficiência do TENS modo convencional como forma promoção de analgesia e seus efeitos na força muscular respiratória.

\section{METODOLOGIA}

Trata-se de um ensaio clínico não controlado de corte transversal em indivíduos no período pós-operatório de cirurgia bariátrica, realizado na Fundação Hospitalar São Lucas, na cidade de Cascavel - PR. Para realização deste estudo, foi enviado o projeto ao Comitê de Ética e Pesquisa (CEP) do Centro Universitário da Fundação Assis Gurgacz sendo aprovado em 18 de maio de 2018 e registrado sob o número 2.663.023 (Anexo 1).

A amostra da pesquisa foi composta por 10 indivíduos, de ambos os sexos, que foram sujeitos à realização de cirurgia bariátrica do tipo Capella e com todos os participantes foram empregados o tratamento com a utilização de eletroanalgesia utilizando o TENS. 
A rotina analgésica proposta pelo protocolo da equipe médica que realizou a cirurgia foi mantida com todos os participantes, rotina esta que contava com a utilização dos medicamentos Dipirona e Tramal sendo administrados de horário e também o Dimorf este sendo utilizado a critério do médico plantonista do período.

Como critérios de inclusão foram estabelecidos que os pacientes possuíssem idade mínima de 18 anos e máxima de 50, não apresentassem instabilidade hemodinâmica, estivessem no pós-operatório imediato ou $1^{\circ}$ dia de pós-operatório de cirurgia bariátrica e nível de consciência igual a 15 segundo a escala de coma de Glasgow (Anexo 2). Foram exclusos pacientes que apresentaram nível de consciência menor ou igual a 14 na escala de coma de Glasgow, instabilidade hemodinâmica, contraindicações quanto ao uso do TENS (como presença de marca-passo cardíaco, cardiopatas, dor sem etiologia, epiléticos e gestantes durante primeiro trimestre) e aqueles que optaram por não participar do estudo.

Uma vez preenchido os critérios de inclusão, os participantes foram esclarecidos sobre o procedimento a ser realizado assim como seus benefícios e riscos. Através da assinatura do Termo de Consentimento Livre Esclarecido (TCLE) (Apêndice 1) os pacientes concederam a permissão para a realização da pesquisa.

Antes e após a utilização do TENS os participantes foram sujeitos a uma avaliação (Apêndice 2), que foi realizada através da verificação da pressão arterial com uso do esfigmomanômetro e estetoscópio da marca Premium®, saturação parcial de oxigênio e frequência cardíaca com uso do oxímetro de dedo da marca Bioland®. Também foi realizada a avaliação de sensibilidade tecidual, em que o participante permaneceu de olhos vendados enquanto o examinador realizou palpação do local onde foi feita a aplicação dos eletrodos, além do nível de consciência através da escala de coma de Glasgow. Neste momento ainda, os participantes foram questionados sobre o nível de dor através da Escala Visual Analógica (EVA) (anexo 3) e foram orientados a quantificar seu nível de dor em uma escala de 0 a 10. Através da manovacuômetria foram obtidos os valores da pressão inspiratória máxima (PIMÁX) e pressão expiratória máxima (PEMÁX). A técnica utilizada para a medição destes valores foi a mesma preconizada nas diretrizes para testes de funções pulmonares do Jornal Brasileiro de Pneumologia (2002) e para a medição da força muscular respiratória foi utilizado o Manovacuômetro digital marca Blodalmed modelo MVD 300.

A EVA é uma escala que consiste em uma linha reta, numerada, de dez centímetros, apresentada em uma folha em branco, em que a extremidade esquerda numerada com 0 significa sem dor e a extremidade direita numerada com 10 significa pior dor imaginável (ANDRELLA et al, 2007), foi solicitado para o participante apontar o local que correspondia a seu nível de dor no momento.

A aplicação do TENS foi realizada uma única vez e respeitando um período mínimo de 30 minutos antes da administração medicamentosa, aplicação esta que foi realizada com o aparelho de TENS KLD Endophasys $®$ modelo NMS.0501, utilizando dois canais e 4 (quatro) eletrodos autoadesivos com formato quadrado de tamanho 5 por $5 \mathrm{~cm}$.

Para a realização do procedimento os pacientes foram orientados para que permanecessem sentados. Os parâmetros utilizados para aplicação do TENS foram os mesmos preconizados por Morimoto et al, (2009), sendo utilizada a corrente convencional com frequência de $80 \mathrm{~Hz}$ e $\mathrm{T}=200 \mathrm{us}$, pulso bipolar assimétrico. A intensidade da corrente foi modulada conforme a sensibilidade umbral de cada paciente. Os eletrodos foram posicionados a $3 \mathrm{~cm}$ da incisão cirúrgica nos quatro 
quadrantes e os canais utilizados foram posicionados de forma cruzada para que a corrente se concentrasse sobre a incisão.

Os dados obtidos foram analisados com a utilização da plataforma SPSS 20.0 (Statistical Package for the Social Sciences). Para analisar a distribuição da amostra foi utilizado o teste de Shapiro-Wilk, que apresentou valores superiores a 0,05 indicando assim a apresentação dos dados em média e desvio padrão. O teste $\mathrm{T}$ de Student para amostras em pares foi realizado, considerando níveis de significância menores que 0,05 .

\section{RESULTADOS E DISCUSSÃO}

Foram avaliados neste estudo 10 pacientes, sendo 1 (10\%) do sexo masculino e $9(90 \%)$ do sexo feminino. A média de idade dos participantes foi de 39,6 $\pm 11,787$. Com relação ao tipo de cirurgia os 10 participantes (100\%) realizaram cirurgia do tipo Capella e mantiveram a rotina de analgesia padrão. Os dados referentes à avaliação da pressão arterial, saturação parcial de oxigênio e frequência cardíaca não apresentaram diferenças estatísticas entre os dois momentos conforme apresentado na tabela 1.

Tabela 1. Avaliação dos sinais vitais

\begin{tabular}{ccccccc}
\hline & PA inicial & PA final & Spo2 inicial & Spo2 final & FC inicial & FC final \\
\hline & $130 / 80$ & $130 / 80$ & $98 \%$ & $99 \%$ & 91 & 82 \\
& $150 / 90$ & $150 / 90$ & $91 \%$ & $93 \%$ & 71 & 86 \\
& $150 / 100$ & $150 / 100$ & $98 \%$ & $98 \%$ & 75 & 79 \\
& $140 / 90$ & $140 / 90$ & $93 \%$ & $91 \%$ & 64 & 70 \\
& $150 / 90$ & $150 / 90$ & $96 \%$ & $96 \%$ & 90 & 90 \\
& $140 / 80$ & $140 / 80$ & $94 \%$ & $91 \%$ & 88 & 82 \\
& $140 / 80$ & $140 / 80$ & $90 \%$ & $93 \%$ & 120 & 107 \\
& $140 / 90$ & $140 / 90$ & $97 \%$ & $97 \%$ & 77 & 80 \\
& $140 / 90$ & $140 / 90$ & $91 \%$ & $93 \%$ & 75 & 89 \\
Dédia & $120 / 70$ & $120 / 70$ & $92 \%$ & $95 \%$ & 81 & 90 \\
\hline
\end{tabular}

Legenda: PA = Pressão Arterial; Spo2 = Saturação parcial de oxigênio; FC= Frequência Cardíaca. Valores de PA apresentados em mmhg; Valores de FC apresentados em BPM (batimentos por minuto), D.P - Desvio Padrão.

De acordo com a prova de manovacuômetria, os valores obtidos não apresentaram diferenças estatísticas entre os dois momentos. Já em relação à avaliação da dor, sugere-se uma redução do quadro de dor no pós-intervenção $(2,2 \pm 1,98)$ quando comparado com os valores no pré-intervenção $(5,6 \pm 1,57)$ com $\mathrm{P}=0,000$. Os resultados dos testes de força muscular e os valores da EVA podem ser visualizados na tabela 2.

Sabe-se que em cirúrgicas abdominais, os pacientes estão sujeitos a apresentarem disfunções relacionadas à sua função pulmonar, principalmente evidenciada com a força muscular respiratória, isso devido ao quadro de dor ou até mesmo pela incisão cirúrgica, que de forma direta afeta a integridade da musculatura (COSTA et al, 2009). Evidências mostram que a disfunção diafragmática está entre 
os principais responsáveis por complicações relacionadas à função pulmonar (LAWRENCE et al, 2006).

O fator dor, avaliado no presente estudo, mostrou-se concordante com esses fatos tendo em vista que os 10 participantes avaliados apresentaram níveis elevados de dor, mesmo mantendo a rotina analgésica pós-operatória.

Tabela 2. Resultados testes de força muscular e escala de dor

\begin{tabular}{lllll}
\hline Variável & Momento & Média & D.P & P \\
\hline E.V.A & Pré-intervenção & 5,60 & 1,57 & \\
\multirow{2}{*}{ PIMAX } & Pós-intervenção & 2,20 & 1,98 & $0,000^{*}$ \\
& Pré-intervenção & $-93,00$ & $-35,60$ & \\
PEMAX & Pós-intervenção & $-96,00$ & $-35,33$ & 0,081 \\
& Pré-intervenção & 71,00 & 31,78 & \\
& Pós-intervenção & 85,00 & 35,97 & 0,116 \\
\hline
\end{tabular}

Nota: *Valores significativos - Teste T para amostras em pares; D.P = Desvio Padrão.

Imle (2003) e Fraga (2004) afirmam que a presença de dor em pacientes no pósoperatório de laparotomia é frequente e o expõem a riscos desnecessários. Lee (2008) em estudo realizado com 66 pacientes no pós-operatório imediato observou que $72,7 \%$ dos participantes apresentavam níveis elevados de dor.

Para Braz et al, (2009) a presença de uma equipe de fisioterapia em ambiente hospitalar é de extrema importância e desempenha um papel chave atuando através da prevenção e reabilitação de disfunções pulmonares e/ou motoras, utilizando diversas técnicas, contribuindo para uma redução na taxa de mortalidade e menor tempo de internamento hospitalar.

Com os níveis elevados de dor apresentados no pós-operatório e as repercussões pulmonares que isso acarreta, observa-se a necessidade de intervenções visando à diminuição do quadro álgico para uma melhor efetividade das técnicas fisioterapêuticas, para que então se promova uma melhora na função respiratória, isso é confirmado por Silva et al, (2010) que em estudo realizado com pacientes que realizaram cirurgia de laparotomia e foram submetidos a um protocolo de exercícios respiratórios demonstraram um aumento de 42,10\% no nível de dor após a intervenção fisioterápica.

Dentre os recursos utilizados pela fisioterapia, o TENS, vem sendo utilizado como forma de redução do quadro de dor no pós-operatório, mostrando-se um método acessível, de baixo custo e eficaz (MORIMOTO et al, 2009). A corrente produz analgesia por agir na comporta da dor conforme explicado por Melzack e Wall, (1965). Bjordal et al, (2003) realizou uma meta-análise, onde 21 ensaios clínicos randomizados foram avaliados, chegando à conclusão que com o uso do TENS os pacientes apresentaram uma redução de $26,5 \%$ em média o consumo de medicação analgésica nas primeiras 72 horas de pós-operatório.

O efeito da terapia com uso de TENS é obtido através da estimulação de fibras responsáveis pela transmissão ao encéfalo de estímulos proprioceptivos ascendentes (TONELLA et al, 2006), além de ser embasado pela "Teoria das Comportas" (MELZACK \& WALL, 1965).

Melo et al, (2005) realizou um estudo onde foi utilizado o TENS modo convencional em pacientes no pós operatório de cesariana e observou-se uma redução significativa no nível de dor corroborando com os resultados obtidos neste estudo onde o nível de dor avaliado através da EVA sugeriu uma redução de $5,6 \pm 1,57$ 
no momento inicial para 2,2 $\pm 1,98$ quando avaliados após a aplicação da eletroanalgesia.

Gregorini et al, (2010) em ensaio clinico prospectivo randomizado com pacientes em pós-operatório de cirurgia cardíaca, observou uma melhora em relação ao nível de dor em repouso de $40 \%$ e à tosse de $42,9 \%$ no grupo que recebeu tratamento com TENS comparado ao grupo placebo.

Em estudo realizado por Paisani et al, (2005) com pacientes em pré e pósoperatório de gastroplastia, com avaliação da força muscular respiratória observou uma diminuição de $51 \%$ na pimáx e $39 \%$ na pemáx no primeiro dia de pós-operatório, isso pode ser observado pois segundo Barbalho-Moulim et al, (2009) no pósoperatório de cirurgia bariátrica, mesmo por via laparoscópica, ocorre um comprometimento da função pulmonar, visto pela diminuição da mobilidade toracoabdominal e diafragmática, além da redução dos volumes pulmonares.

Os dados obtidos no estudo em questão com relação à força muscular respiratória, não apresentaram valores estatisticamente significativos com $p>0,05$, quando comparados entre os dois momentos (PIMÁX PRÉ 93 $\pm 35,6$ e pós 96 $\pm 35,33$ $\mathrm{p}=0,081$, PEMÁX PRÉ $71 \pm 31,78$ e pós $85 \pm 35,97 \mathrm{p}=0,116$ ), porém observa-se um discreto aumento nos valores obtidos, mesmo com apenas 30 minutos de aplicação da corrente, dados esses semelhantes aos descritos por Vieira et al, (2011) em estudo com pacientes no período pós operatório de gastroplastia, os valores de PIMÁX e PEMÁX não se diferenciaram entre o grupo controle e grupo placebo após aplicação da corrente TENS.

\section{CONSIDERAÇÕES FINAIS}

Conclui-se que o TENS convencional é uma ferramenta eficaz no alívio da dor pós-operatória mesmo com apenas a realização de uma única aplicação, entretanto não demonstrou influência nos valores relacionados à força muscular respiratória.

\section{REFERÊNCIAS}

ANDRELLA. G. Q et al. Estudo comparativo entre duas escalas e a aplicação em doentes. Goiânia, v. $34, \quad$ n. 1/2, p. 21-34, jan./fev. 2007. http://dx.doi.org/10.18224/est.v34i1.305

BARBALHO-MOULIM M. C, MIGUEL G. P. S, FORTI E. M. P, COSTA D. Comparação entre inspirometria de incentivo e pressão positiva expiratória na função pulmonar após cirurgia bariátrica. Fisioterapia e Pesquisa, São Paulo, v.16, n.2, p.166-72, abr./jun. 2009. http://dx.doi.org/10.1590/S1809-29502009000200013

BATTISTELLI, C. Sociedade Brasileira de Cirurgia Bariátrica e Metabólica. Número de cirurgias bariátricas no Brasil aumenta 46,7\%, 11 jul. 2018. Disponível em: <https://www.sbcbm.org.br/numero-de-cirurgias-bariatricas-no-brasil-aumenta-467/> Acesso em: 24 set. 2018.

BENEDETTI F, AMANZIO M, CASADIO C, CAVALLO A, CIANCI R, GIOBBE R, ET $A L$. Control of postoperative pain by transcutaneous electrical nerve stimulation after thoracic operation. Ann Thorac Surg. 1997; 63 (3): 773-6.

https://doi.org/10.1016/S0003-4975(96)01249-0 
BJORDAL JM, JOHNSON MI, LJUNGGREEN AE - Transcutaneous electrical nerve stimulation (tens) can reduce postoperative analgesic consumption. A meta-analysis with assessment of optimal treatment parameters for postoperative pain. Eur j pain, 2003;7:181-188. https://doi.org/10.1016/S1090-3801(02)00098-8

BUCHWALD H, WILLIAMS SE. Bariatric surgery worldwide 2003. Obes Surg. 004;14(9):1157-64. BRAZ PE, MARTINS JO, JUNIOR GV. Atuação do fisioterapeuta nas unidades de terapia intensiva da cidade de Anápolis. Anuário da Produção Acadêmica Docente Vol. III, №. 4, Ano 2009.

COSTA D. FORTI E. M. P , BARBALHO-MOULIM M. C, RASERA-JUNIOR I. Estudo dos volumes pulmonares e da mobilidade toracoabdominal de portadoras de obesidade mórbida, submetidas à cirurgia bariátrica, tratadas com duas diferentes técnicas de fisioterapia. Rev Bras Fisioter, São Carlos, v. 13, n. 4, p. 294-300, jul./ago. 2009.

http://dx.doi.org/10.1590/S1413-35552009005000041

CIPRIANO. G; CARVALHO. A. C. C; BERNARDELLI. G. F; PERES. P. T. Short-term transcutaneous electrical nerve stimulation after cardiac surgery: effect on pain, pulmonary function and electrical muscle activity. Interact Cardio Vasc Surg. 2008; 7: 539-43.

http://dx.doi.org/10.5935/1678-9741.20120012

ERDOGAN.M; ERDOGAN. A; ERBIL.N; KARAKAYA. H; DEMIRCAN A. Prospective, randomized, placebo-controlled study of the effect of TENS on postthoracotomy pain and pulmonary function. World J Surg. 2005; 29: 1563-70.

https://doi.org/10.1007/s00268-005-7934-6

FANDIÑO J, BENCHIMOL A. K,. COUTINHO W.F; APPOLINÁRIO J.C. Cirurgia bariátrica: aspectos clínico-cirúrgicos e psiquiátricos. Rev. psiquiatr. Rio Gd. Sul vol.26 no.1 Porto Alegre Jan./Apr. 2004. http://dx.doi.org/10.1590/S0101$\underline{81082004000100007}$

FRAGA G. P, MANTOVANI M, MAGNA L. A. Índices de trauma em pacientes submetidos à laparotomia. Rev Col Bras Cir. 2004;31(5):299-306.

http://dx.doi.org/10.1590/S0100-69912004000500006

GREGORINI, C, JUNIOR G. C, AQUINO L. M, BRANCO J. N. R, BERNADELLI G. F. Estimulação elétrica nervosa transcutânea de curta duração no pós-operatório de cirurgia cardíaca. Arquivos Brasileiros de Cardiologia, São Paulo, v. 94, n. 3, mar. 2010.

http://dx.doi.org/10.1590/S0066-782X2010000300011

IMLE P.C. Fisioterapia em pacientes com problemas cardíacos, torácicos ou abdominais após cirurgia ou trauma. In: Irwin SI, Teckln JS. Fisioterapia cardiopulmonar. 3 ed. São Paulo: Manole; 2003.p.375-403.

LAWRENCE V. A, CORNELL J. E, SMETANA G. W, American Collage of Physicians. Strategies to reduce postoperative pulmonary complications after noncardiothoracic 
surgery: systematic review for the american college of physicians. Ann Intern Med. 2006;144(8):596-608. DOI: 10.7326/0003-4819-144-8-200604180-00011

LEE F. V. S. Avaliação da dor pós-operatória. (Trabalho de conclusão de curso) Florianópolis: Curso de Graduação em Medicina, Universidade Federal de Santa Catarina; 2008.

MASCARENHAS, J. Q. P, GUEDES, Alexis Dourado. Fisioterapia em pacientes submetidos à cirurgia abdominal alta. Revisão de literatura. Pós Graduação em Fisioterapia Hospitalar 2014.

MELO P. G, MOLINERO P. V. R, DIAS R. O, MATTEI K. Estimulação elétrica nervosa transcutânea (TENS) no pós-operatório de cesariana. Rev. bras. fisioter. Vol. 10, No. 2 (2006), 219-224.

MELZACK R, WALL P - Pain mechanisms: a new theory. Science, 1965;150:971979.

MORGAN C. R, SANTOS F. S. Estudo da estimulação elétrica nervosa transcutânea (TENS) nível sensório para efeito de analgesia em pacientes com osteoartrose de joelho. Fisioter. Mov., Curitiba, v. 24, n. 4, p. 637-646, out./dez. 2011.

http://dx.doi.org/10.1590/S0103-51502011000400007

MORIMOTO, H.C, YONEKURA M.Y e LIEBANO R.E. Estimulação elétrica nervosa transcutânea nas modalidades convencional e acupuntura na dor induzida pelo frio. Fisioterapia e Pesquisa, São Paulo, v.16, n.2, p.148-54, abr./jun. 2009.

http://dx.doi.org/10.1590/S1809-29502009000200010

OGUNNAIKE. O. JONES. S. B. JONES. D. B. PROVOST. D. WHITTEN. C. W. Anesthetic Considerations for Bariatric Surgery. Anesth Analg. 2002;95:1793-1805. DOI: 10.1097/00000539-200212000-00061

ORIA H. E, MOOREHEAD M. K. Bariatric Analysis and Reportig Outcome System (BAROS) Obes Surg 1998;8:487-99.

https://doi.org/10.1381/096089298765554043

PAISANI. D. M, VEGATO. L. D. C, FARESIN. S. M. Volumes, capacidades pulmonares e força muscular respiratória no pós-operatório de gastroplastia. Jornal Brasileiro de Pneumologia. 31(2) - Mar/Abr de 2005.

http://dx.doi.org/10.1590/S1806-37132005000200007

RAO V.R, WOLF S.L, GERSH M.R. Examination of electrode placements and stimulating parameters in treating chronic pain with conventional transcutaneous electrical nerve stimulation (TENS). Pain. 1981;11(1):37-47.

DOI: $10.1016 / 0304-3959(81) 90137-8$

SILVA F. A, LOPES T. M, DUARTE J, MEDEIROS R. F. Tratamento fisioterapêutico no pós-operatório de laparotomia. J Health Sci Inst. 2010; 28(4):341-4.

SEGAL A, FANDIÑO J. Indicações e contra-indicações para realização das operações bariátricas. Rev. Bras. Psiquiatr. vol.24 suppl.3 São Paulo Dec. 2002.

DOI: $10.35984 /$ fjh.v2i1.114

FAG Journal of Health - ISSN 2674-550X, 2020, v.2, n.1, p. 54 


\section{http://dx.doi.org/10.1590/S1516-44462002000700015}

SOUZA R. B. Pressões respiratórias estáticas máximas. J Pneumol 28(Supl 3) outubro de 2002.

TONELLA R. M, ARAÚJO S, SILVA A. M. Estimulação Elétrica Nervosa Transcutânea no Alívio da Dor Pós-Operatória Relacionada com Procedimentos Fisioterapêuticos em Pacientes Submetidos a Intervenções Cirúrgicas Abdominais. Revista Brasileira de Anestesiologia Vol. 56, No 6, Novembro-Dezembro, 2006.

http://dx.doi.org/10.1590/S0034-70942006000600007

TREVISAN, M. E. SOARES, J. C. RONDINEL, T. Z. Efeitos de duas técnicas de incentivo respiratório na mobilidade toracoabdominal após cirurgia abdominal alta. Fisioterapia e Pesquisa, São Paulo, v.17, n.4, p.322-6, out/dez. 2010. http://dx.doi.org/10.1590/S1809-29502010000400007

TRIBIOLI RA. Análise critica atual sobre a TENS envolvendo parâmetros de estimulação para o controle da dor [dissertação de mestrado]. Ribeirão Preto: Universidade de São Paulo, Curso de Bioengenharia, Programa de Pós-Graduação Interunidades em Bioengenharia; 2003.

DOI:10.11606/D.82.2003.tde-10092003-094654

VIEIRA B. O. A; BARBOSA R. R. A; BRITO J. S; ARAÚJO I; FERRARI F. Estimulação elétrica nervosa transcutânea no alívio da dor no pós-operatório de gastroplastia. Nov/2011. Disponível em: < https://interfisio.com.br/estimulacao-eletrica-nervosatranscutanea-no-alivio-da-dor-no-pos-operatorio-de-gastroplastia/> Acesso em 01 out 2018.

http://dx.doi.org/10.5935/1678-9741.20110049

WORLD HEALTH ORGANIZATION. Obesity: prevent - ing and managing the global epidemic. Gene - va: World Health Organization; 2000. (WHO Technical Report Series, 894). 